\title{
Prognostic roles of mitochondrial transcription termination factors in non-small cell lung cancer
}

\author{
SHUANGYAN SUN ${ }^{1 *}$, CHUNJIAO WU ${ }^{2 *}$, CHANGLIANG YANG $^{2}$, JIAN CHEN $^{3}$, \\ XIU WANG ${ }^{3}$, YINGJI NAN ${ }^{1}$, ZHICHENG HUANG ${ }^{1}$ and LIXIA MA ${ }^{2}$ \\ Departments of ${ }^{1}$ Radiology, ${ }^{2}$ Thoracic Oncology and ${ }^{3}$ Interventional Radiology, \\ Jilin Province Cancer Hospital, Changchun, Jilin 130012, P.R. China
}

Received April 29, 2018; Accepted May 2, 2019

DOI: $10.3892 / 01.2019 .10680$

\begin{abstract}
Mitochondrial transcription termination factors (MTERFs) regulate mitochondrial gene transcription and metabolism in numerous types of cells. Previous studies have indicated that MTERFs serve pivotal roles in the pathogenesis of various cancer types. However, the expression and prognostic roles of MTERFs in patients with non-small cell lung cancer (NSCLC) remain elusive. The present study investigated the gene alteration frequency and expression level using Gene Expression Omnibus datasets and reverse transcription-quantitative polymerase chain reaction, and evaluated the prognostic roles of MTERFs in patients with NSCLC using the Kaplan-Meier plotter database. In human lung cancer tissues, it was observed that the mRNA levels of MTERF1, 2, 3 and 4 were positively associated with the copy number of these genes. The mRNA expression levels of MTERF1 and 3 were significantly increased in NSCLC tissues compared with adjacent non-tumor tissues; however, the mRNA expression of MTERF2 was significantly decreased in NSCLC tissues. High mRNA expression levels of MTERF1,
\end{abstract}

Correspondence to: Dr Zhicheng Huang, Department of Radiology, Jilin Province Cancer Hospital, 1018 Lake Road, Changchun, Jilin 130012, P.R. China

E-mail: 33088401@qq.com

Dr Lixia Ma, Department of Thoracic Oncology, Jilin Province Cancer Hospital, 1018 Lake Road, Changchun, Jilin 130012, P.R. China

E-mail: lixiama1234567@163.com

*Contributed equally

Abbreviations: MTERF, mitochondrial transcription termination factor; NSCLC, non-small cell lung cancer; LC, lung cancer; LUAD, lung adenocarcinoma; LUSC, lung squamous cell carcinoma; KM plotter, Kaplan-Meier plotter; HR, hazard ratio; OS, overall survival; TGCA, The Cancer Genome Atlas; GEO, Gene Expression Omnibus; mtDNA, mitochondrial DNA; NSUN4, NOP2/Sun RNA methyltransferase family member 4

Key words: mitochondrial transcription termination factor, non-small cell lung cancer, Kaplan-Meier plotter, prognostic roles
2, 3 and 4 were strongly associated with an improved overall survival rate (OS) in patients with lung adenocarcinoma. Additionally, high mRNA expression levels of MTERF1, 2, 3 and 4 were also strongly associated with an improved OS of patients with NSCLC in the earlier stages of disease (stage I) or patients with negative surgical margins. These results indicate the critical prognostic values of MTERF expression levels in NSCLC. The findings of the present study may be beneficial for understanding the molecular biology mechanism of NSCLC and for generating effective therapeutic approaches for patients with NSCLC.

\section{Introduction}

Mitochondria are recognized as the primary sites of aerobic oxidation of metabolic fuels in eukaryotic cells (1). It is well established that mitochondria provide the majority of cellular energy in the form of ATP through electron transport chains and an oxidative phosphorylation system $(2,3)$. According to a previous study, mitochondria contribute to various pivotal cell functions, including nitrogen metabolism, pyruvate and fatty acid oxidation and heme biosynthesis (4). Mitochondria have their own genomic DNA, making them unique among eukaryotic extranuclear organelles; and the mitochondrial genome is a circular double-stranded DNA (5).

The mitochondrial transcription termination factor (MTERF) family members serve important roles in mitochondrial gene expression (6). The MTERF family consists of diverse members, including MTERF1, 2, 3 and $4(7,8)$. MTERFs, located in the mitochondria, contain a modular architecture based on repetitions of a 30 amino acid module (8). A previous study indicated that MTERFs control mitochondrial DNA (mtDNA) replication and exert important functions in mitochondria transcription termination and initiation (9). The differences in mitochondrial gene organization have been demonstrated to be associated with a number of functions (8). Among the MTERF family members, MTERF1 is the canonical mitochondrial transcription terminator and is able to modulate the expression of mitochondrial genes by preventing L-strand transcription interference within mtDNA (8). However, MTERF1 is dispensable for ribosomal RNA gene transcription regulation (10). A previous investigation also revealed that defects in MTERF1 binding 
may lead to mitochondrial diseases, including Kearns-Sayre syndrome (11). In post-transcriptional modification patterns of mitochondrial genes, a mitochondrial encephalomyopathy, lactic acidosis, and stroke-like episodes (MELAS) mutation in the mtDNA binding-site for the transcription termination factor was revealed to cause defects in protein-synthesis in respiration; however, it does not alter the upstream and downstream mature transcription levels (12). MTERF2 is highly expressed in liver, heart and skeletal muscle cells (13). Previous experiments in mice demonstrated that, when fed on a ketogenic diet (i.e. high fat and low carbohydrate diet), MTERF2-deficient mice developed myopathy and memory deficits (14). In the absence of MTERF2, mtDNA transcription is markedly decreased and a respiratory defect occurs in neurons (14). MTERF3 is another member of the MTERF family and is essential for the development of embryos in mice (15). MTERF4 contributes to the regulation of mitochondrial translation by targeting NOP2/sun RNA methyltransferase family member 4 (NSUN4) to large mitochondrial ribosome, and MTERF4-knockout also leads to mouse embryo death (16). A previous study also provided a novel insight into the association between MTERF4 and $\mathrm{MPP}^{+}$-induced mitochondrial damage (17). In addition, MTERF4 has been hypothesized to be one of the triggering genes for Parkinson's disease, which is induced by an environmental toxin (17). Neurological disorders, including Parkinson's disease and Alzheimer's disease, and also ageing, diabetes and cancer are associated with mitochondrial dysfunction resulting from abnormal variations in mitochondrial gene expression $(18,19)$.

As one of the major causes of mortality worldwide, lung cancer (LC) is primarily characterized as small cell lung cancer and non-small cell lung cancer (NSCLC). Over 80\% of patients with LC are characterized as having NSCLC (19). Typically, NSCLC comprises of lung squamous cell carcinoma (LUSC) and lung adenocarcinoma (LUAD) (20). A number of studies have suggested that mitochondrial functions are involved in the development of LC, particularly mitochondrial dysfunction may lead to an alternation in bioenergetics and genomic instability in LC (21). Additionally, a previous study indicated that mitochondrial fission and fusion are involved in metabolic function, proliferation and cell survival during the progression of LC (22). Recently, various novel approaches and techniques have been used to identify and investigate novel biomarkers and potential molecular mechanism in various types of cancer (23-26), including LC and renal cancer. However, there are $>8.2$ million LC-associated mortalities worldwide each year and the mortality rates of LC remain at unacceptably high levels (20). Inspired by the aforementioned previous studies, the present study aimed to investigate potential biological associations between mitochondrial gene expression and the pathogenesis of NSCLC. MTERFs are understood to regulate mitochondrial functions through modulating the mitochondrial gene transcription and protein expression $(6,9,10)$; however, to the best of our knowledge, there are no reports regarding the prognostic roles of MTERFs in patients with NSCLC. In addition, the role of MTERFs in NSCLC remain elusive; therefore, the present study aimed to investigate the expression and prognostic values of MTERFs in patients with NSCLC.

\section{Materials and methods}

Collection of human lung cancer tissues and ethics statement. Lung cancer tissues and paired adjacent non-tumor normal lung tissues from patients with NSCLC were obtained at the Jilin Province Cancer Hospital (Changchun, China) between January 2012 and December 2012, as described previously (27). The patients included 5 males and 4 females with a mean age of 55.5 years (age range, 45-73 years), A total of 9 tumor and 9 non-tumor tissue samples were collected from these patients using video assisted thoracoscopic (VATS) lobectomy. During surgery, NSCLC and adjacent tissues ( $5 \mathrm{~cm}$ from cancerous tissues) were obtained and subsequently stored at $-80^{\circ} \mathrm{C}$. Pathological data were studied prospectively following surgery. Cancer tissue specimens were diagnosed by pathology, and adjacent tissues were diagnosed as no cancer or inflammatory cell infiltration. All fresh tissues were stored at $-80^{\circ} \mathrm{C}$ for subsequent experiments. The present study was approved by the Ethics Committee of Jilin Province Cancer Hospital. Written informed consent was obtained from all participants prior to enrollment in the present study. The study protocols were performed based on the Declaration of Helsinki.

Reverse transcription-quantitative polymerase chain reaction (RT-qPCR) analysis of gene expression. Total RNA from human lung tissue was extracted using TRIzol ${ }^{\circledR}$ reagent (Invitrogen; Thermo Fisher Scientific, Inc., Waltham, MA, USA). Total RNA $(1 \mu \mathrm{g})$ was reverse-transcribed into cDNA using a reverse transcription kit (iScript ${ }^{\mathrm{TM}}$ Reverse Transcription Supermix for RT-qPCR; Bio-Rad Laboratories, Inc., Hercules, CA, USA). Briefly, the temperature protocol for cDNA synthesis was as follows: $5 \mathrm{~min}$ at $25^{\circ} \mathrm{C}, 20 \mathrm{~min}$ at $46^{\circ} \mathrm{C}$ and $1 \mathrm{~min}$ at $96^{\circ} \mathrm{C}$. RT-qPCR analysis of gene expression was performed using the following primers: MTERF1 forward, 5'-TTGGATGAC TCGATTTTCAGCA-3' and reverse, 5'-GCTGTCGTTTCC TTGCCAT-3'; MTERF2 forward, 5'-TTCAGAAAGATGCGA TCACCTC-3' and reverse, 5'-TCATCGGCACCTAGTTCT TGT-3'; MTERF3 forward, 5'-GCTTTGTCAGCCCAACAG ATA-3' and reverse, 5'-CTTGAGCTAGTAGACTGGGAA CT-3'; MTERF4 forward, 5'-GCCTGTATGGCTAGGCAG AC-3' and reverse, 5'-GGAGGCTGTAGTCAGTTTGCG-3'; and GAPDH forward, 5'-ACAACTTTGGTATCGTGGAAG G-3' and reverse, 5'-GCCATCACGCCACAGTTTC-3'. qPCR analysis was performed using SYBR Premix Ex Taq (Takara Bio, Inc., Otsu, Japan). PCR products were monitored with an Mx3000P QPCR system (Agilent Technologies, Inc., Santa Clara, CA, USA). The thermocycling conditions were as follows: $10 \mathrm{sec}$ at $95^{\circ} \mathrm{C}, 40$ cycles of $5 \mathrm{sec}$ at $95^{\circ} \mathrm{C}$, and $30 \mathrm{sec}$ at $60^{\circ} \mathrm{C}$. The mRNA expression levels were analyzed using the $2^{-\Delta \Delta \mathrm{Cq}}$ method (28) with the Mx3000P QPCR system, and the levels of MTERF1-4 were normalized to the constitutive expression level of GAPDH mRNA.

Gene alteration frequency and mRNA expression in NSCLC from the Gene Expression Omnibus (GEO) database. The present study analyzed the gene alteration frequency of MTERF1-4 in patients with NSCLC from The Cancer Genome Atlas (TCGA) database (http://cancergenome.nih.gov) and the PubMed database (https://www.ncbi.nlm.nih.gov/pubmed) 
A
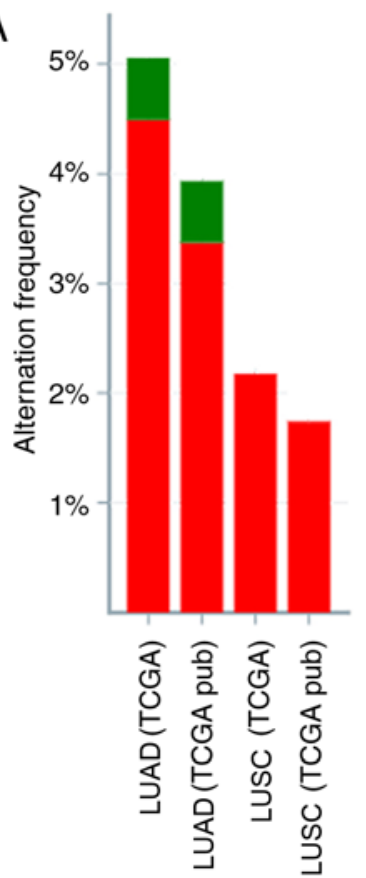

B
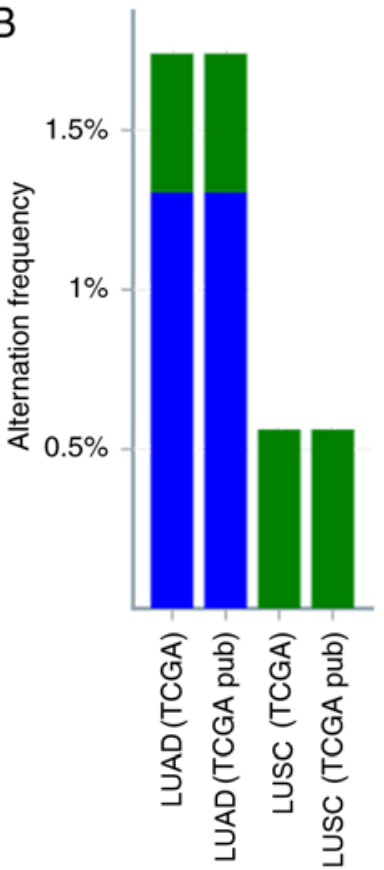

C
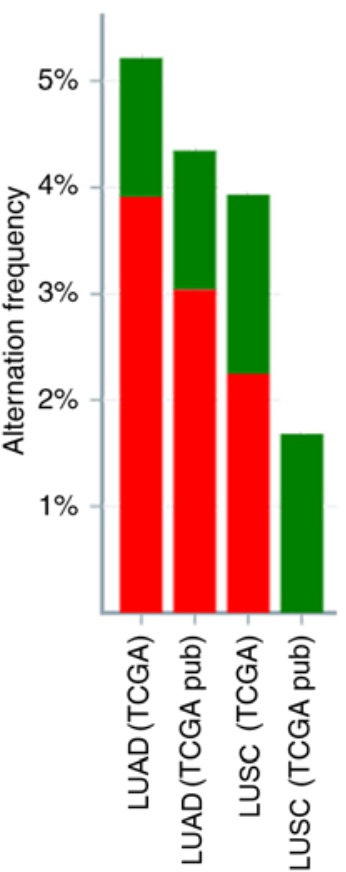

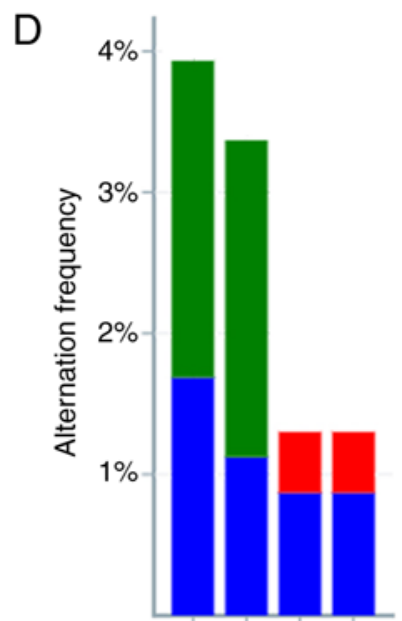

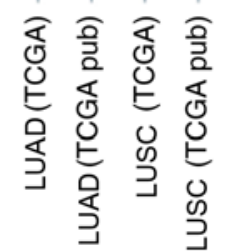

Mutation

Amplification

Deep deletion

Figure 1. Alteration frequency of MTERF1-4 gene expression in NSCLC. The alterations of MTERF1, 2, 3 and 4 gene expressions were visualized using the cBioPortal for Cancer Genomics database. Mutations, deletions and amplifications are presented in different colors. Alteration frequencies of (A) MTERF1, (B) MTERF2, (C) MTERF3 and (D) MTERF4 gene expression in NSCLC according to TCGA database. NSCLC, non-small cell lung cancer; TCGA, The Cancer Genome Atlas; MTERF, mitochondrial transcription termination factor; pub, PubMed; LUAD, lung adenocarcinoma; LUSC, lung squamous cell carcinoma.

using the cBioportal for cancer genomics analysis (http://www. cbioportal.org) (29). The present study also analyzed the mRNA expression levels of MTERF1-4 in NSCLC using the GEO database (http://www.ncbi.nlm.nih.gov/geo). A total of two original datasets (GSE10072 and GSE19804) (30) for patients with LC were downloaded from GEO. The mRNA expression levels of MTERF1-4 in NSCLC samples and adjacent non-tumor tissues were used for subsequent analysis.

Prognostic values of MTERFs in patients with NSCLC. In order to evaluate the prognostic roles of MTERFs in patients with NSCLC, the present study used public databases to analyze the expression of these genes and the overall survival rate (OS) of 1,926 patients with NSCLC, who completed follow-up for 20 years. The data used for analysis were pooled from GEO, TCGA, European Genome-phenome Archive (https://ega.crg. eu/) and PubMed (http://www.pubmed.com)(31). To obtain Kaplan-Meier plots for the patients OS rate, the Kaplan-Meier plotter (KM plotter) database (http://kmplot.com/analysis/index. $\mathrm{php}$ ? $=$ service\&cancer=lung) was used. According to the median expression level of all patients with NSCLC included in the analysis, the patients were divided into two groups: High expression group and low expression group. Hazard ratios (HRs), 95\% confidence intervals (CIs) and log-rank P-values were also calculated from the database. $\mathrm{P}<0.05$ was considered to indicate a statistically significant difference (32). The following Affymetrix IDs were valid: 204871_at (MTERF1), 225346_at (MTERF2), 219363_s_at (MTERF3) and 1557965_at (MTERF4).
Statistical analysis. RT-qPCR assays were analyzed from a minimum of three independent experiments. Data were analyzed using a paired, two-tailed Student' t-test or one-way ANOVA using GraphPad Prism version 6 (GraphPad Software, Inc., La Jolla, CA, USA). Data are presented as the mean \pm standard error of the mean (32). $\mathrm{P}<0.05$ was considered to indicate a statistically significant difference.

\section{Results}

MTERFs gene alteration frequency in NSCLC. The present study initially analyzed the gene alteration frequency of MTERFs in NSCLC tissues using the cBioportal database. As presented in Fig. 1, high amplification rates of MTERF1 and MTERF3 were observed in NSCLC (Fig. 1A and C). MTERF2 was revealed to exhibit a high deep deletion rate in patients with LUAD and a high mutation rate in patients with LUSC (Fig. 1B). In addition, MTERF4 exhibited a high mutation and high deep deletion rate in patients with LUSC (Fig. 1D). The mutation of all MTERF genes primarily occurred in the same region, which contains three leucine zipper sequences (data not shown); and the leucine zipper sequences are critical for the enzyme functions (33).

Expression of MTERFs in NSCLC. The present study subsequently analyzed the mRNA expression of MTERFs and its correlation with the copy numbers using cBioportal for cancer genomics analysis (http://www.cbioportal.org) as previously 
A

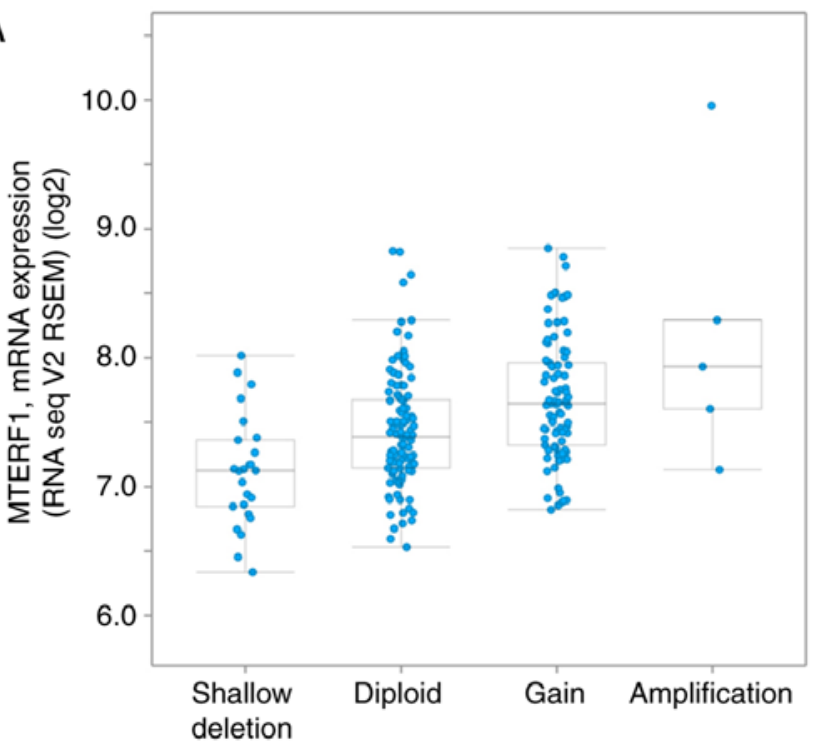

MTERF1, putative copy-number alternations

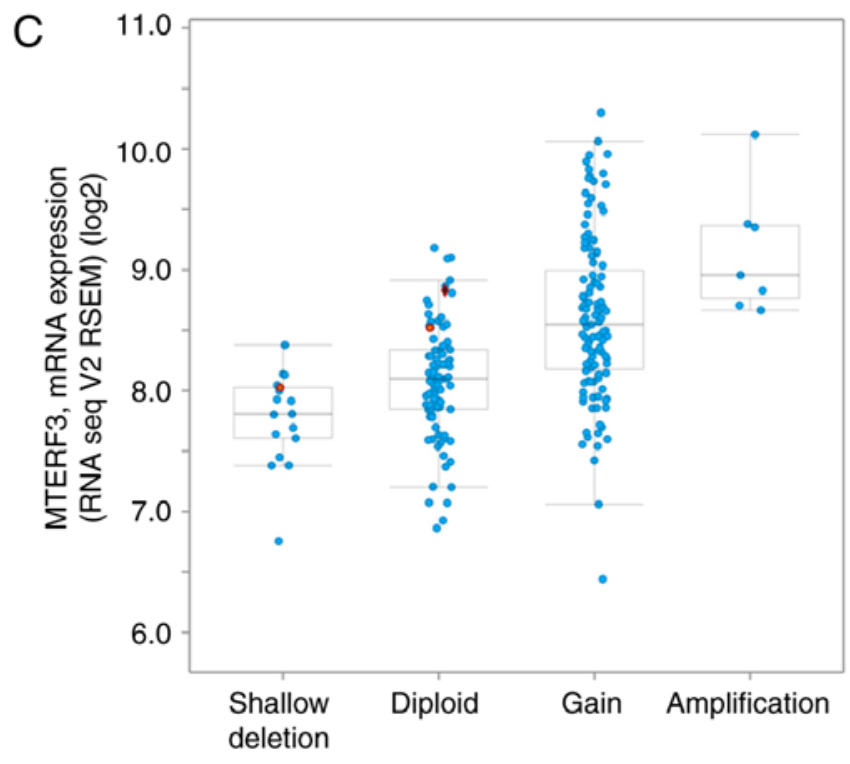

MTERF3,

putative copy-number alternations
B
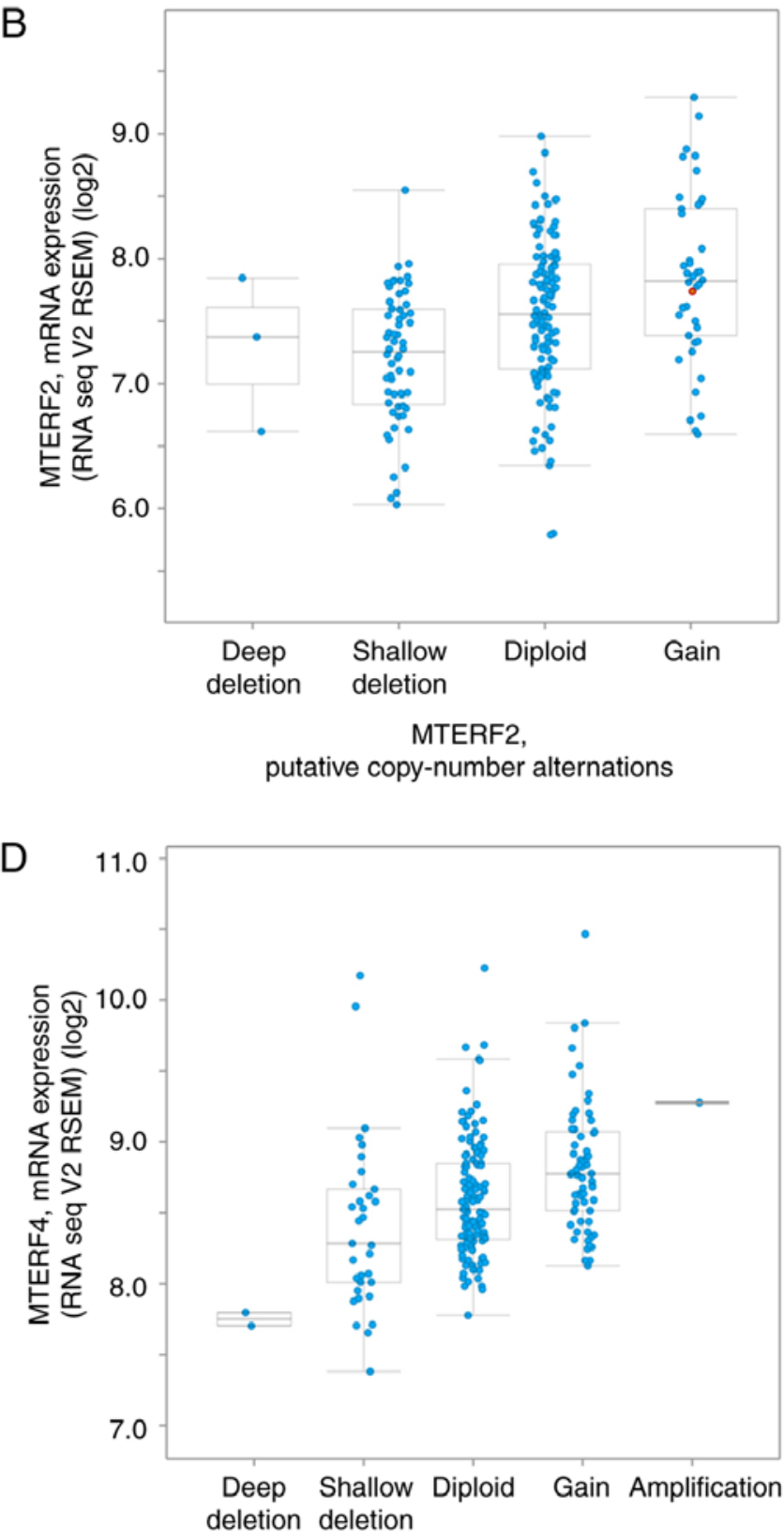

MTERF4,

putative copy-number alternations

Figure 2. Correlations of MTERF1-4 mRNA levels with copy number alterations in non-small cell lung cancer. (A) MTERF1, (B) MTERF2, (C) MTERF3 and (D) MTERF4. MTERF, mitochondrial transcription termination factor.

described (28). As illustrated in Fig. 2, the mRNA levels of MTERF1, 2, 3 and 4 were positively associated with the copy number of these genes in patients with LUAD (Fig. 2). To investigate the mRNA expression of MTERFs in NSCLC and normal tissues, the raw data was retrieved by searching terms 'GSE\#19804' and 'GSE\#10072' from the GEO dataset. Additionally, the present study analyzed the mRNA expression of MTERF1-4 by RT-qPCR in the fresh isolated NSCLC tissues, which were collected at Jilin Province Cancer Hospital in 2012, as described previously (27). The subsequent analysis indicated that the mRNA expression levels of MTERF1 (Fig. 3A and B) and MTERF3 (Fig. 3D and E) were significantly higher $(\mathrm{P}<0.001)$ in NSCLC tissues compared with paired adjacent non-tumor tissues from micro-array analysis. The mRNA expression of MTERF2 was significantly decreased in lung tumor tissue $(\mathrm{P}<0.05$; Fig. $3 \mathrm{C})$. However, the expression of MTERF4 was not significantly changed in NSCLC tissues when compared with adjacent non-tumor tissues (Fig. 3F and G). Additionally, qPCR analysis in isolated lung cancer tissue observed the increase of MTERF1 (Fig. 4A) and MTERF3 (Fig. 4C) mRNA expression $(\mathrm{P}<0.05)$, the decrease of MTERF2 (Fig. 4B) mRNA expression $(\mathrm{P}<0.05)$ by comparing with the non-tumor tissues. However, the expression MTERF4 in the fresh isolated tumor tissue has no different by comparing with that from adjacent non-tumor tissues (Fig. 4D). 
A

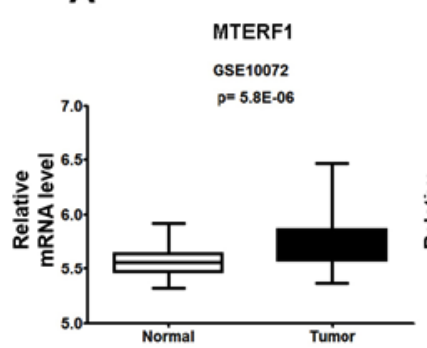

B

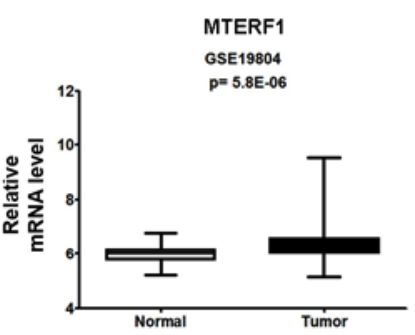

C

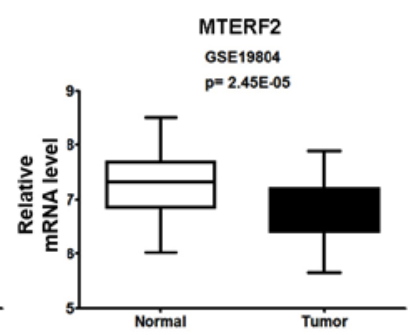

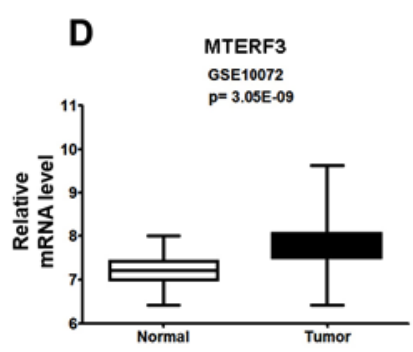
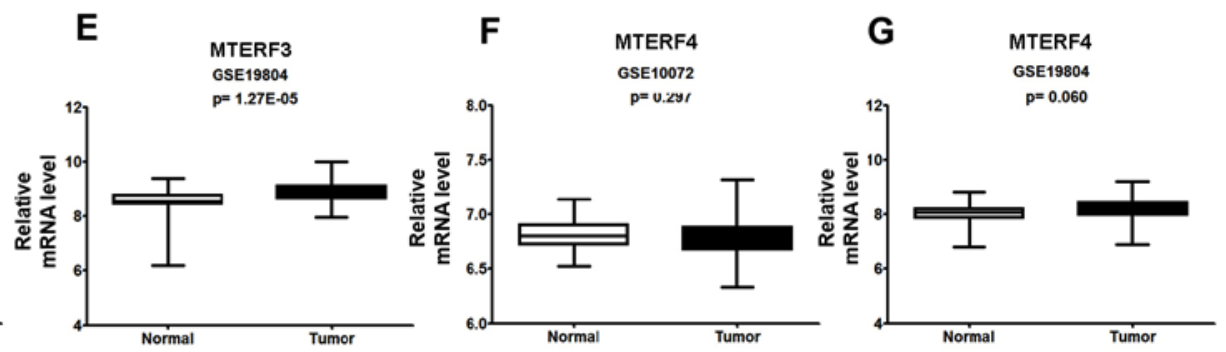

Figure 3. Expression of MTERFs in normal and tumor tissues from human patients. MTERF1-4 mRNA expression levels in lung tumors were compared with that in the adjacent normal tissue. Expression of MTERF1 in human lung cancer tissues and normal tissues in (A) GSE10072 and (B) GSE19804. (C) Expression of MTERF2 in human lung cancer tissues and normal tissues in GSE19804. Expression of MTERF3 in human lung cancer tissues and normal tissues in (D) GSE10072 and (E) GSE19804. Expression of MTERF4 in human lung cancer tissues and normal tissues in (F) GSE10072 and (G) GSE19804. Data are presented as the mean \pm standard error of the mean. MTERF, mitochondrial transcription termination factor.
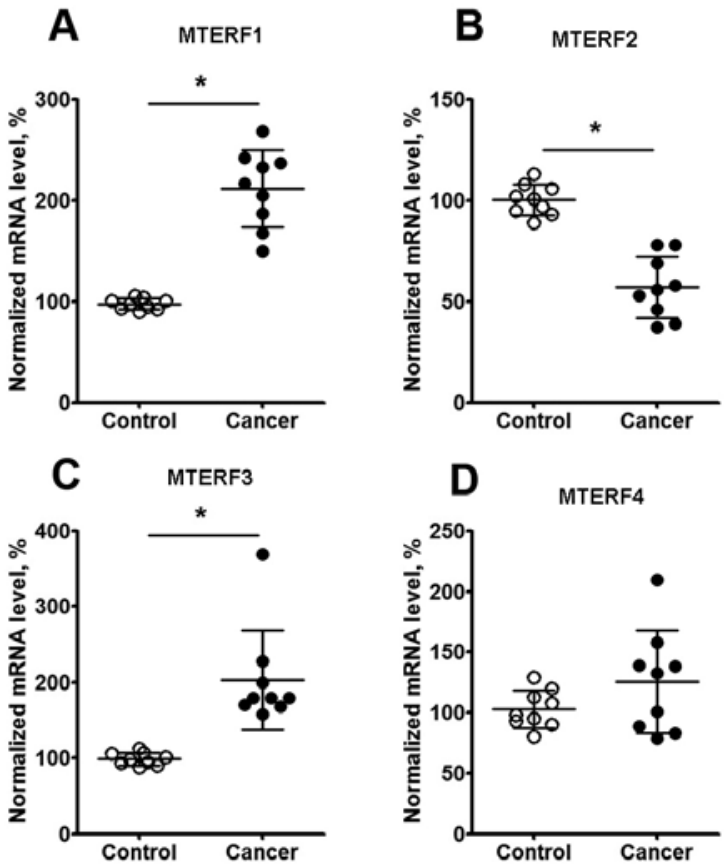

Figure 4. RT-qPCR analysis of the mRNA expression levels of MTERF1-4 in normal and NSCLC tumor tissues. mRNA expression levels of (A) MTERF1, (B) MTERF2, (C) MTERF3 and (D) MTERF4 in cancer tissue and adjacent normal tissues from patients with NSCLC. The mRNA expression levels were analyzed by RT-qPCR and normalized to the expression of GAPDH. The normalized expression levels are presented as the mean \pm standard error of the mean. ${ }^{*} \mathrm{P}<0.05$. RT-qPCR, reverse transcription-quantitative polymerase chain reaction; MTERF, mitochondrial transcription termination factor; NSCLC, non-small cell lung cancer.

Associations between MTERF expression levels and clinicopathological characteristics of patients with NSCLC. The present study used KM plotter to evaluate the prognostic value of MTERFs in patients with NSCLC. High MTERF1 mRNA expression was significantly associated with improved OS rate in patients with LUAD ( $\mathrm{n}=720$; HR, 0.52; 95\% CI, 0.39-0.70; $\mathrm{P}=8 \times 10^{-6}$; Fig. 5A). For MTERF2, the survival curves were generated for patients with LUAD ( $n=673$; Fig. 5B). Notably, high mRNA expression of MTERF2 was significantly associated with improved OS rate in patients with LUAD $(n=673$; HR 0.48; 95\% CI, 0.36-0.66; $\mathrm{P}=1.9 \times 10^{-6}$; Fig. 5B). Furthermore, high MTERF3 mRNA expression was significantly associated with improved OS rate for patients with LUAD $(n=591$; HR, 0.69; 95\% CI, 0.54-0.88; P=0.0028; Fig. 5C). For MTERF4, a high mRNA expression level was significantly associated with an improved OS rate for patients with LUAD ( $n=673$; HR, 0.51; 95\% CI, 0.38-0.68; $\mathrm{P}=2.4 \times 10^{-6}$; Fig. 5D). However, for patients with LUSC, higher mRNA expression levels of MTERF1 (n=524; HR, 1.1; 95\% CI, 0.87-1.40; P=0.42; Fig. 6A), MTERF3 (HR, 1.23; 95\% CI, 0.95-1.58; P=0.11; Fig. 6C) and MTERF4 (HR, 1.21; 95\% CI, 0.84-1.74; P=0.31; Fig. 6D) were not associated with better OS. Higher mRNA expression of MTERF2 was also marginally associated with improved OS in patients with LUSC for MTERF2 $(\mathrm{n}=271$; HR, 1.5; 95\% CI, 1.05-2.13; $\mathrm{P}=0.024$; Fig. 6B).

To further assess the association of MTERFs with other clinicopathological factors, the present study determined the associations of OS rate with the patients' sex (Table I), smoking status (Table II), different clinical stages (Table III) and surgical margins (Table IV). As presented in Table I, high expression levels of MTERF2 and 4, but not MTERF1 and 3, were significantly associated with improved OS in both male and female patients with NSCLC. As presented in Table II, the expression levels of MTERF1 and 2 were significantly associated with the OS in patients with NSCLC who smoked and never smoked. However, a high expression of MTERF3 was significantly associated with a higher OS in patients who never smoked (HR, 0.25; 95\% CI, 0.09-0.69; P=0.0037) but not in patients who smoked. Additionally, a high expression 
A

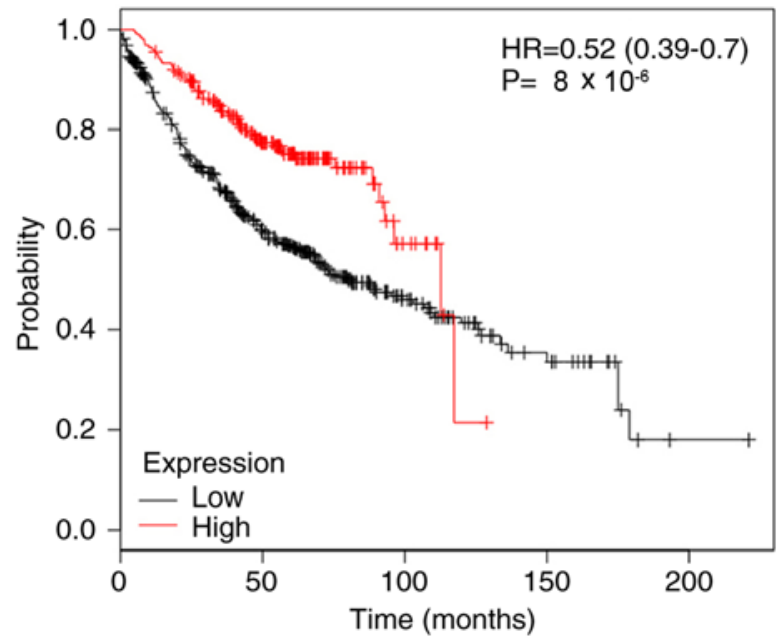

Low $498 \quad 229 \quad 59 \quad 19 \quad 1$

High $222 \quad 119 \quad 10 \quad 0 \quad 0$

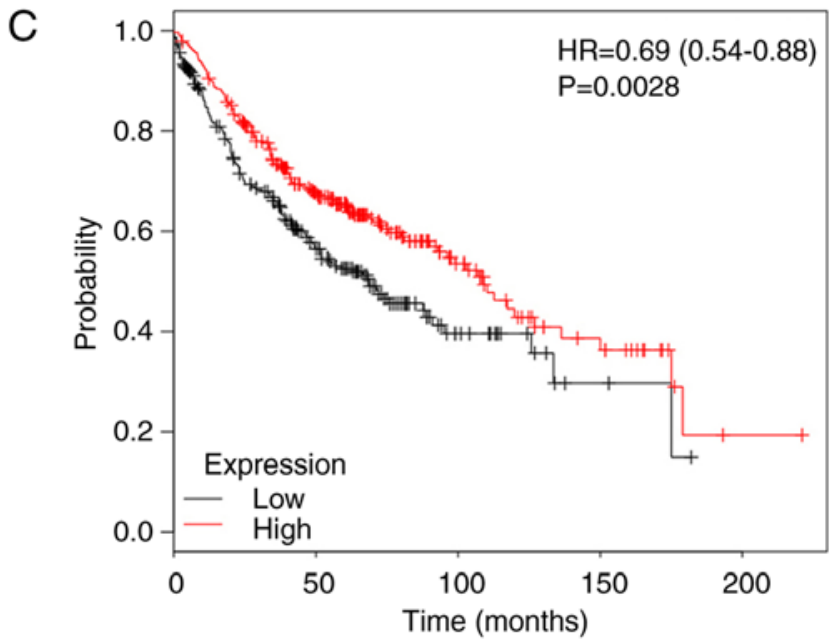

Low $306 \quad 125$

High $285 \quad 156$

203

$44 \quad 16$

B

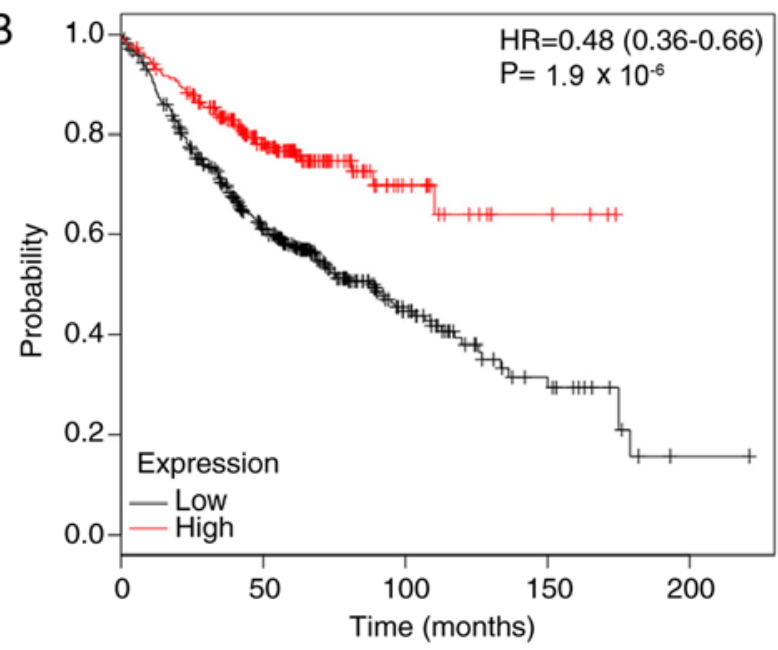

$\begin{array}{lllcl}\text { Low 457 } & 225 & 52 & 15 & 1 \\ \text { High 216 } & 120 & 17 & 4 & 0\end{array}$

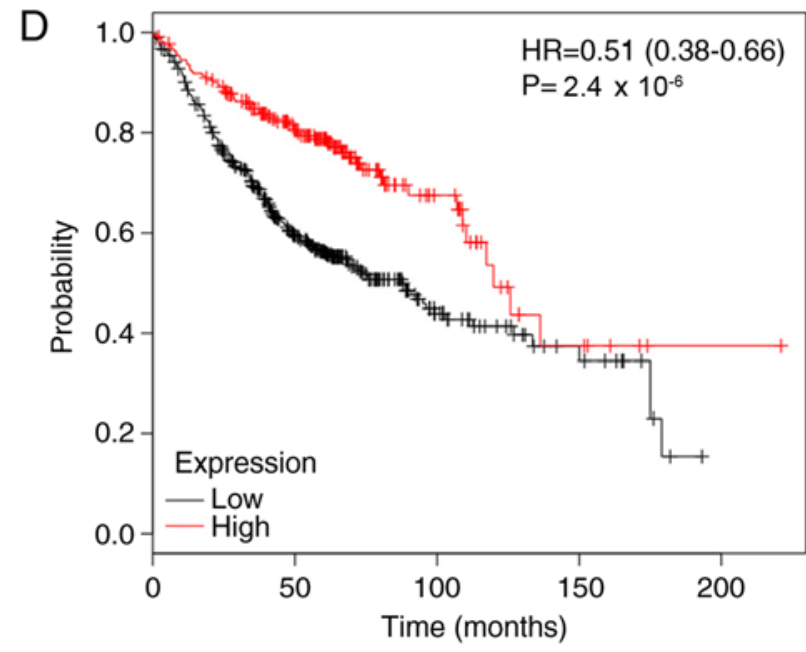

$\begin{array}{ccccc}\text { Low } 450 & 204 & 43 & 13 & 0 \\ \text { High } 223 & 141 & 26 & 6 & 1\end{array}$

Figure 5. Prognostic value of MTERF1-4 expression in patients with LUAD. Survival curves for patients with LUAD according to the expression level of (A) MTERF1, (B) MTERF2, (C) MTERF3 and (D) MTERF4. MTERF, mitochondrial transcription termination factor; LUAD, lung adenocarcinoma.

of MTERF4 was identified to be significantly associated with OS of patients who smoked (HR, 0.33; 95\% CI, 0.19-0.59; $\mathrm{P}=4.1 \times 10^{-5}$ ) but not patients who never smoked. As presented in Table III, low MTERF1-4 mRNA expression was associated with a poorer OS rate for patients with NSCLC in earlier stages. Particularly, for patients in stage I, lower expression levels of MTERF1 $(\mathrm{P}=0.0006)$, MTERF2 $\left(\mathrm{P}=1.3 \times 10^{-5}\right)$, MTERF3 $\left(\mathrm{P}=2.4 \times 10^{-8}\right)$ and MTERF4 $\left(\mathrm{P}=2.4 \times 10^{-8}\right)$ were significantly associated with a worse OS rates (Table III). For patients with stage II tumors, the lower expression levels of MTERF1 $(\mathrm{P}=0.00053)$ and MTERF3 $(\mathrm{P}=0.0063)$, but not the MTERF2 and MTERF4 were significantly associated with worse OS rates (Table III). For patients in stage III, only the lower expression of MTERF2 ( $\mathrm{P}=0.032)$ but not MTERF1, MTEF3 and MTERF4 was significantly associated with a worse OS rates (Table III). As presented in Table IV, a low mRNA expression of MTERF1-4 was associated with a worse OS in patients with negative surgical margins.

\section{Discussion}

The MTERF family consists of four members, termed MTERF1-4, which have been identified in plants but not in fungi (8). MTERFs contain leucine zipper-like heptads, localize to the mitochondria, and are involved in mitochondrial gene transcription and protein synthesis (8). Among the four MTERFs, MTERF1 and 2 are uniquely expressed in vertebrates (34). In humans, all MTERFs are expressed in lung tissue (35). In general, a number of studies have indicated that MTERFs bind to mtDNA promoter regions and regulate mitochondrial gene expression $(6,7,9,10)$. Previous studies have suggested that the roles of MTERFs are likely more complex than just regulating mitochondrial gene expression $(9,10)$. Human MTERF1 is the most well characterized family member of the MTERFs, and is a mitochondrial protein consisting of 342 amino acids (8). MTERF1 modulates mitochondrial gene replication and regulates mitochondrial gene expression by 
Table I. Association of MTERF1-4 expression with the overall survival rate of patients with NSCLC of different sexes.

\begin{tabular}{|c|c|c|c|c|c|}
\hline Gene & Sex & Cases, n & HR & $95 \%$ CI & P-value \\
\hline MTERF1 & Male & 1,00 & 0.88 & $0.75-1.04$ & 0.14 \\
\hline MTERF1 & Female & 715 & 0.74 & $0.59-0.93$ & 0.01 \\
\hline MTERF2 & Male & 659 & 0.77 & $0.61-0.97$ & 0.027 \\
\hline MTERF2 & Female & 375 & 0.4 & $0.25-0.64$ & $7.4 \times 10^{-5}$ \\
\hline MTERF3 & Male & 1,103 & 1.19 & $1.00-1.41$ & 0.055 \\
\hline MTERF3 & Female & 619 & 1.25 & $0.93-1.68$ & 0.14 \\
\hline MTERF4 & Male & 659 & 0.64 & $0.50-0.82$ & 0.0032 \\
\hline MTERF4 & Female & 375 & 0.45 & $0.32-0.63$ & $2.0 \times 10^{-6}$ \\
\hline
\end{tabular}

MTERF, mitochondrial transcription termination factor; HR, hazard ratio; CI, confidence interval; NSCLC, non-small cell lung cancer.

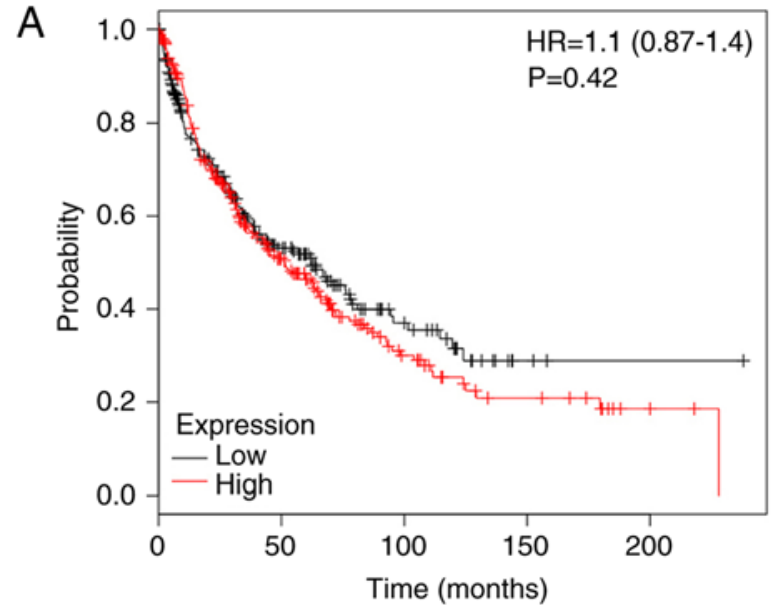

$\begin{array}{llccc}\text { Low } 256 & 84 & 25 & 3 & 1 \\ \text { High } 268 & 95 & 29 & 12 & 3\end{array}$

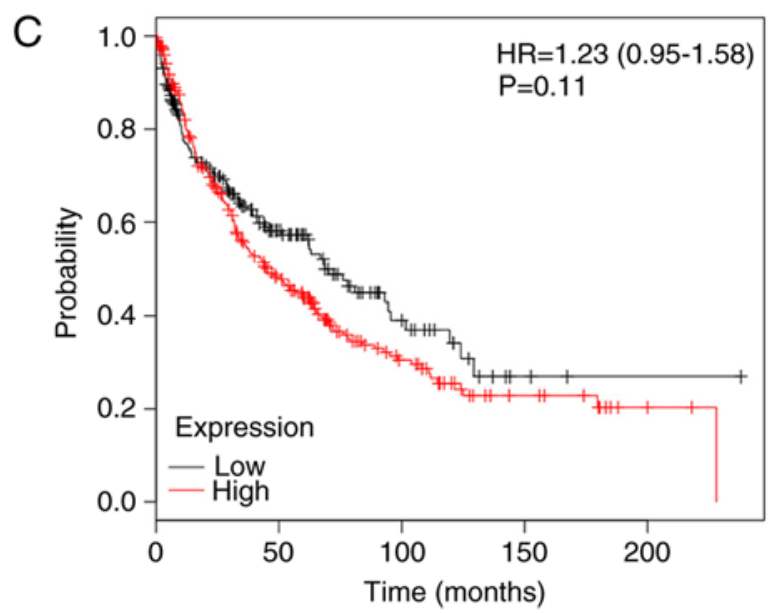

Low $215 \quad 71$

High $277 \quad 101 \quad 34 \quad 12 \quad 30$

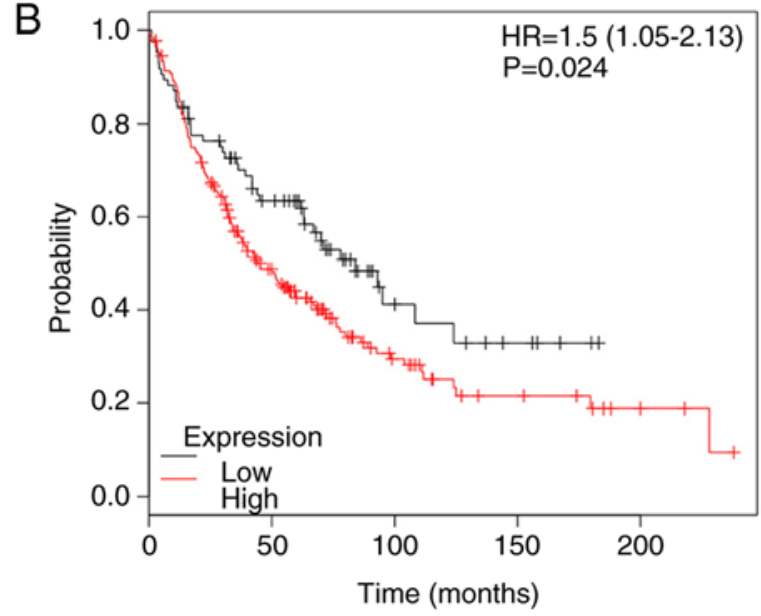

$\begin{array}{ccccc}\text { Low } 85 & 47 & 11 & 5 & 0 \\ \text { High } 186 & 73 & 23 & 10 & 4\end{array}$

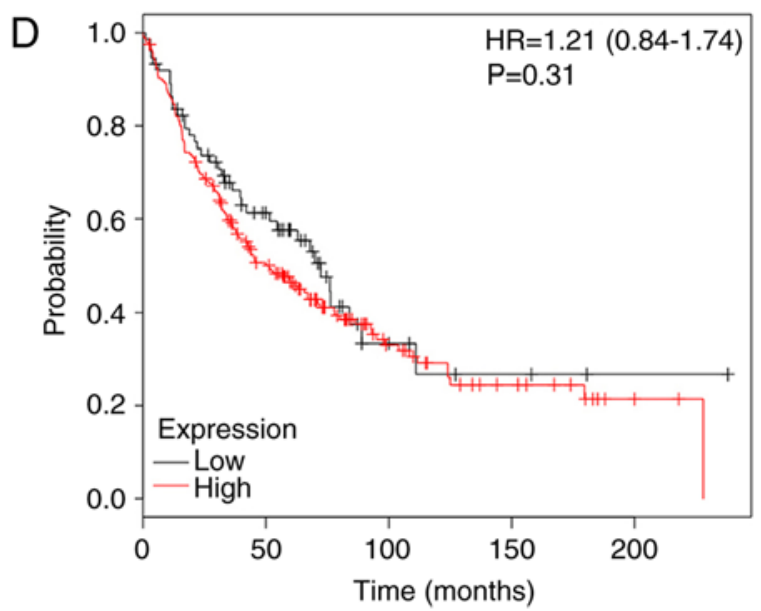

Low $74 \quad 34 \quad 7 \quad 3 \quad 3 \quad 1$

$\begin{array}{lllll}\text { High } 197 & 86 & 27 & 12 & 3\end{array}$

Figure 6. Prognostic value of MTERF1-4 expression in patients with LUSC. Survival curves for patients with LUSC according to the expression level of (A) MTERF1,(B) MTERF2, (C) MTERF3 and (D) MTERF4. MTERF, mitochondrial transcription termination factor; LUSC, lung squamous cell carcinoma.

arresting mitochondrial RNA polymerase progression (36). The pathogenic MELAS mutation of MTERF1 has been demonstrated to attenuate the ability of transcription termination in vitro $(11,37)$. Additionally, MTERF2 is highly expressed in heart, liver and skeletal muscle cells (14), and was demonstrated to bind to the promoter region of mitochondrial 
Table II. Association of MTERF1-4 expression with the overall survival rate of patients with NSCLC with different smoking statuses.

\begin{tabular}{llcccc}
\hline Gene & Smoking status & Cases, $\mathrm{n}$ & HR & 95\% CI & P-value \\
\hline MTERF1 & Smoked & 820 & 0.66 & $0.51-0.86$ & 0.0022 \\
MTERF1 & Never smoked & 205 & 0.24 & $0.13-0.45$ & $1.3 \times 10^{-6}$ \\
MTERF2 & Smoked & 300 & 0.38 & $0.22-0.63$ & $1 \times 10^{-4}$ \\
MTERF2 & Never smoked & 141 & 0.25 & $0.11-0.59$ & 0.0064 \\
MTERF3 & Smoked & 702 & 1.19 & $0.95-1.50$ & 0.12 \\
MTERF3 & Never smoked & 152 & 0.25 & $0.09-0.69$ & 0.0037 \\
MTERF4 & Smoked & 300 & 0.33 & $0.19-0.59$ & $4.1 \times 10^{-5}$ \\
MTERF3 & Never smoked & 141 & 0.36 & $0.16-0.84$ & 0.13 \\
\hline
\end{tabular}

MTERF, mitochondrial transcription termination factor; HR, hazard ratio; CI, confidence interval; NSCLC, non-small cell lung cancer.

Table III. Association of MTERF1-4 expression with the overall survival rate of patients with NSCLC of different clinical stages.

\begin{tabular}{lccccc}
\hline Gene & Clinical stage & Cases, $\mathrm{n}$ & HR & $95 \%$ CI & P-value \\
\hline MTERF1 & I & 577 & 0.63 & $0.48-0.82$ & 0.0006 \\
MTERF1 & II & 244 & 0.49 & $0.33-0.74$ & 0.00053 \\
MTERF1 & III & 70 & 1.4 & $0.81-2.42$ & 0.23 \\
MTERF2 & I & 449 & 0.44 & $0.30-0.64$ & $1.3 \times 10^{-5}$ \\
MTERF2 & II & 161 & 1.21 & $0.77-1.90$ & 0.41 \\
MTERF2 & III & 44 & 0.48 & $0.24-0.95$ & 0.032 \\
MTERF3 & I & 476 & 0.76 & $0.57-1.01$ & $2.4 \times 10^{-8}$ \\
MTERF3 & II & 215 & 0.58 & $0.39-0.86$ & 0.0063 \\
MTERF3 & III & 53 & 0.71 & $0.38-1.32$ & 0.27 \\
MTERF4 & I & 449 & 0.36 & $0.25-0.52$ & $2.4 \times 10^{-8}$ \\
MTERF4 & II & 161 & 0.62 & $0.36-1.06$ & 0.078 \\
MTERF4 & III & 44 & 2.09 & $0.94-4.67$ & 0.066 \\
\hline
\end{tabular}

MTERF, mitochondrial transcription termination factor; HR, hazard ratio; CI, confidence interval; NSCLC, non-small cell lung cancer.

Table IV. Association of MTERF1-4 expression with the overall survival rate of patients with NSCLC with negative surgical margins.

\begin{tabular}{lcllc}
\hline Gene & Cases, $\mathrm{n}$ & HR & $95 \%$ CI & P-value \\
\hline MTERF1 & 726 & 0.38 & $0.28-0.51$ & $3.7 \times 10^{-11}$ \\
MTERF2 & 204 & 0.2 & $0.09-0.42$ & $2.6 \times 10^{-6}$ \\
MTERF3 & 615 & 0.63 & $0.46-0.86$ & 0.036 \\
MTERF4 & 204 & 0.29 & $0.14-0.61$ & 0.00052 \\
\hline
\end{tabular}

MTERF, mitochondrial transcription termination factor; HR, hazard ratio; CI, confidence interval; NSCLC, non-small cell lung cancer.

genes and to regulate the initiation of mitochondrial gene transcription $(6,7,9,10)$. MTERF2-knockout mice exhibit myopathy and memory deficits, which may be due to decreased mtDNA transcription (38). In addition, the loss of MTERF2 in mice results in decreased oxidative phosphorylation complexes and causes a respiratory defect (14). Similarly, MTERF3 is also involved in mitochondrial biogenesis in cells of humans and other vertebrates (39). Knockout of MTERF3 in mice results in embryo mortality (40), which indicates that MTERF3 functions as an essential factor in mouse embryonic development. Furthermore, tissue specific inactivation of MTERF3 in the heart significantly decreased the life span of mice, and may affect heart and skeletal muscle functions by regulating mitochondrial functions (15). MTERF4 has a common fold similar to MTERF1 and MTERF3, which contains positively charged surfaces and may be beneficial for nucleic acid interaction (16). In human cells, 3D crystal structure analysis indicated that MTERF4 is required for mitochondrial ribosomal biogenesis and translation via forming a stoichiometric complex with NSUN4 (16). In a human neuroblast cell line, knockdown of MTERF4 increased the $\mathrm{MPP}^{+}$-induced mitochondrial dysfunction, which may be due to increased mtDNA transcription and translation levels (17). Similar to MTERF3, MTERF4-knockdown in mice also induces embryo mortality $(6,40)$. Furthermore, the loss of MTERF4 in the mouse heart also disrupts the transfer RNA pool; however, it 
increases steady-state levels of mtDNA transcripts (17). To the best of our knowledge, until now, the roles of MTERFs in the pathogenesis of NSCLC have not been investigated.

Mitochondrial functions are essential for eukaryotic cells $(3,4)$. Defects in mitochondrial gene expression are associated with numerous diseases, including LC (1). Mitochondrial gene mutations are common in cancer development and may regulate mitochondrial metabolism (1). The results of the present study provide insight into the roles of MTERFs in NSCLC pathogenesis and suggest an association between mitochondrial gene transcription and LC. The present study investigated the expression and prognostic roles of MTERFs in patients with NSCLC, and the results indicated that the expression levels of MTERF family members were significantly altered in NSCLC tissue. Notably, high expression levels of MTERF1, 2, 3 and 4 were significantly associated with an improved OS for patients with LUAD, and patients with early stage NSCLC. These results indicate that the expression levels of MTERFs may exhibit critical prognostic values for patients with NSCLC; therefore, MTERFs may be used as early biomarkers for predicting the OS of patients with NSCLC. In summary, the present study provides novel insights that may promote further investigations regarding the pathogenesis of NSCLC, and suggests a novel therapeutic strategy of NSCLC via targeting mitochondrial transcription. The present study only focuses on the bioinformatic analysis, and this conclusion may require additional biological experiments in order to accurately identify the potential underlying molecular mechanism.

\section{Acknowledgements}

Not applicable.

\section{Funding}

No funding was received.

\section{Availability of data and materials}

The datasets used and/or analyzed during the present study are available from the corresponding author on reasonable request.

\section{Authors' contributions}

SS, CW, ZH and LM conceived the study and wrote and revised the manuscript. $\mathrm{CY}$ and JC analyzed the gene expression in lung tissues from patients. SS, CW and LM reviewed, collected and analyzed the data. XW, YN, ZH and LM designed the study and acquired the data. All authors contributed to the writing of the manuscript. All authors read and approved the final manuscript.

\section{Ethics approval and consent to participate}

All patients gave their full consent to participate in the present study, and a written consent form was obtained from each patient. The research protocol was approved by the Institutional Review Board of Jilin Province Cancer Hospital.

\section{Patient consent for publication}

A written consent form was obtained from each patient.

\section{Competing interests}

The authors declare that they have no competing interests.

\section{References}

1. Baffy G: Mitochondrial uncoupling in cancer cells: Liabilities and opportunities. Biochim Biophys Acta Bioenerg 1858: 655-664, 2017.

2. Balaban RS: Regulation of oxidative phosphorylation in the mammalian cell. Am J Physiol 258: C377-C389, 1990.

3. Hatefi Y: The mitochondrial electron transport and oxidative phosphorylation system. Annu Rev Biochem 54: 1015-1069, 1985.

4. McCommis KS and Finck BN: Mitochondrial pyruvate transport: A historical perspective and future research directions. Biochem J 466: 443-454, 2015.

5. Gilkerson R, Bravo L, Garcia I, Gaytan N, Herrera A Maldonado A and Quintanilla B: The mitochondrial nucleoid: Integrating mitochondrial DNA into cellular homeostasis. Cold Spring Harb Perspect Biol 5: a011080, 2013.

6. Kleine $\mathrm{T}$ and Leister D: Emerging functions of mammalian and plant mTERFs. Biochim Biophys Acta 1847: 786-797, 2015.

7. Kleine T: Arabidopsis thaliana mTERF proteins: Evolution and functional classification. Front Plant Sci 3: 233, 2012.

8. Roberti M, Polosa PL, Bruni F, Manzari C, Deceglie S, Gadaleta MN and Cantatore P: The MTERF family proteins: Mitochondrial transcription regulators and beyond. Biochim Biophys Acta 1787: 303-311, 2009.

9. Guja KE and Garcia-Diaz M: Hitting the brakes: Termination of mitochondrial transcription. Biochim Biophys Acta 1819: 939-947, 2012

10. Terzioglu M, Ruzzenente B, Harmel J, Mourier A, Jemt E, López MD, Kukat C, Stewart JB, Wibom R, Meharg C, et al: MTERF1 binds mtDNA to prevent transcriptional interference at the light-strand promoter but is dispensable for rRNA gene transcription regulation. Cell Metab 17: 618-626, 2013.

11. Yakubovskaya E, Mejia E, Byrnes J, Hambardjieva E and Garcia-Diaz M: Helix unwinding and base flipping enable human MTERF1 to terminate mitochondrial transcription. Cell 141: 982-993, 2010.

12. Chomyn A, Martinuzzi A, Yoneda M, Daga A, Hurko O, Johns D, Lai ST, Nonaka I, Angelini C and Attardi G: MELAS mutation in mtDNA binding site for transcription termination factor causes defects in protein synthesis and in respiration but no change in levels of upstream and downstream mature transcripts. Proc Natl Acad Sci USA 89: 4221-4225, 1992.

13. Chen Y, Zhou G, Yu M, He Y, Tang W, Lai J, He J, Liu W and Tan D: Cloning and functional analysis of human mTERFL encoding a novel mitochondrial transcription termination factor-like protein. Biochem Biophys Res Commun 337: 1112-1118, 2005.

14. Wenz T, Luca C, Torraco A and Moraes CT: MTERF2 regulates oxidative phosphorylation by modulating mtDNA transcription. Cell Metab 9: 499-511, 2009.

15. Park CB, Asin-Cayuela J, Camara Y, Shi Y, Pellegrini M, Gaspari M, Wibom R, Hultenby K, Erdjument-Bromage H, Tempst $\mathrm{P}$, et al: MTERF3 is a negative regulator of mammalian mtDNA transcription. Cell 130: 273-285, 2007.

16. Camara Y, Asin-Cayuela J, Park CB, Metodiev MD, Shi Y, Ruzzenente B, Kukat C, Habermann B, Wibom R, Hultenby $\mathrm{K}$, et al: MTERF4 regulates translation by targeting the methyltransferase NSUN4 to the mammalian mitochondrial ribosome. Cell Metab 13: 527-539, 2011.

17. Ye X, Han Y, Zhang L, Liu W and Zuo J: MTERF4 regulates the mitochondrial dysfunction induced by MPP(+) in SH-SY5Y cells. Biochem Biophys Res Commun 464: 214-220, 2015.

18. Wallace DC: A mitochondrial paradigm of metabolic and degenerative diseases, aging, and cancer: A dawn for evolutionary medicine. Annu Rev Genet 39: 359-407, 2005.

19. Larsson NG: Somatic mitochondrial DNA mutations in mammalian aging. Annu Rev Biochem 79: 683-706, 2010. 
20. Keith RL and Miller YE: Lung cancer chemoprevention: Current status and future prospects. Nat Rev Clin Oncol 10: 334-343, 2013.

21. Roberts ER and Thomas KJ: The role of mitochondria in the development and progression of lung cancer. Comput Struct Biotechnol J 6: e201303019, 2013.

22. Lennon FE and Salgia R: Mitochondrial dynamics: Biology and therapy in lung cancer. Expert Opin Investig Drugs 23: 675-692, 2014.

23. Hori Y, Otomura N, Nishida A, Nishiura M, Umeno M, Suetake I and Kikuchi K: Synthetic-molecule/protein hybrid probe with fluorogenic switch for live-cell imaging of DNA methylation. J Am Chem Soc 140: 1686-1690, 2018.

24. Wang H, Feng Z, Del Signore SJ, Rodal AA and Xu B: Active probes for imaging membrane dynamics of live cells with high spatial and temporal resolution over extended time scales and areas. J Am Chem Soc 140: 3505-3509, 2018.

25. Liu LJ, Wang W, Huang SY, Hong Y, Li G, Lin S, Tian J, Cai Z, Wang HD, Ma DL and Leung CH: Inhibition of the Ras/Raf interaction and repression of renal cancer xenografts in vivo by an enantiomeric iridium(iii) metal-based compound. Chem Sci 8: 4756-4763, 2017.

26. Vellaisamy K, Li G, Ko CN, Zhong HJ, Fatima S, Kwan HY, Wong CY, Kwong WJ, Tan W, Leung CH and Ma DL: Cell imaging of dopamine receptor using agonist labeling iridium(iii) complex. Chem Sci 9: 1119-1125, 2017.

27. Huang Z, Yang C, Sun S, Nan Y, Lang Z, Wang X, Zhao J and Liu Y: Heat shock protein 27, a novel regulator of transforming growth factor $\beta$ induced resistance to cisplatin in a549 cell. Pharmacology 100: 283-291, 2017.

28. Livak KJ and Schmittgen TD: Analysis of relative gene expression data using real-time quantitative PCR and the 2(-Delta Delta C(T)) method. Methods 25: 402-408, 2001

29. Cerami E, Gao J, Dogrusoz U, Gross BE, Sumer SO, Aksoy BA, Jacobsen A, Byrne CJ, Heuer ML, Larsson E, et al: The cBio cancer genomics portal: An open platform for exploring multidimensional cancer genomics data. Cancer Discov 2: 401-404, 2012.

30. Landi MT, Dracheva T, Rotunno M, Figueroa JD, Liu H, Dasgupta A, Mann FE, Fukuoka J, Hames M, Bergen AW, et al: Gene expression signature of cigarette smoking and its role in lung adenocarcinoma development and survival. PLoS One 3: e1651, 2008.

31. Győrffy B, Surowiak P, Budczies J and Lánczky A: Online survival analysis software to assess the prognostic value of biomarkers using transcriptomic data in non-small-cell lung cancer. PLoS One 8: e82241, 2013.
32. Huang LS, Mathew B, Li H, Zhao Y, Ma SF, Noth I, Reddy SP, Harijith A, Usatyuk PV, Berdyshev EV, et al: The mitochondrial cardiolipin remodeling enzyme lysocardiolipin acyltransferase is a novel target in pulmonary fibrosis. Am J Respir Crit Care Med 189: 1402-1415, 2014.

33. Fernandez-Silva P, Martinez-Azorin F, Micol V and Attardi G: The human mitochondrial transcription termination factor (mTERF) is a multizipper protein but binds to DNA as a monomer, with evidence pointing to intramolecular leucine zipper interactions. EMBO J 16: 1066-1079, 1997.

34. Wobbe L and Nixon PJ: The mTERF protein MOC1 terminates mitochondrial DNA transcription in the unicellular green alga Chlamydomonas reinhardtii. Nucleic Acids Res 41: 6553-6567, 2013.

35. Xu Q, Zhang F, He H, Xu S, Li K, Liu S, Li Y and Wu Q: Expression profile of mouse Mterfd2, a novel component of the mitochondrial transcription termination factor (MTERF) family. Genes Genet Syst 86: 269-275, 2011.

36. Arnold JJ, Smidansky ED, Moustafa IM and Cameron CE: Human mitochondrial RNA polymerase: Structure-function, mechanism and inhibition. Biochim Biophys Acta 1819: 948-960, 2012.

37. Hess JF, Parisi MA, Bennett JL and Clayton DA: Impairment of mitochondrial transcription termination by a point mutation associated with the MELAS subgroup of mitochondrial encephalomyopathies. Nature 351: 236-239, 1991.

38. Wredenberg A, Lagouge M, Bratic A, Metodiev MD, Spåhr H, Mourier A, Freyer C, Ruzzenente B, Tain L, Grönke S, et al: MTERF3 regulates mitochondrial ribosome biogenesis in invertebrates and mammals. PLoS Genet 9: e1003178, 2013.

39. Roberti M, Bruni F, Loguercio Polosa P, Manzari C, Gadaleta MN and Cantatore P: MTERF3, the most conserved member of the mTERF-family, is a modular factor involved in mitochondrial protein synthesis. Biochim Biophys Acta 1757: 1199-1206, 2006.

40. Peralta S, Wang X and Moraes CT: Mitochondrial transcription: Lessons from mouse models. Biochim Biophys Acta 1819: 961-969, 2012.

c) (i) $\ominus$ This work is licensed under a Creative Commons Attribution-NonCommercial-NoDerivatives 4.0 International (CC BY-NC-ND 4.0) License. 